Fecha de recepción: marzo 2020 Fecha de aceptación: abril 2020 Versión final: mayo 2020

\section{ALACINC y la construcción de la identidad latinoamericana en Nueva Zelanda: Un ejercicio de descolonización ${ }^{1}$}

Felipe Forero ${ }^{(1)}$

\begin{abstract}
Resumen: Aotearoa Nueva Zelanda es uno de los ejemplos donde la implementación de políticas multiculturales ha contribuido en el proceso de descolonización partiendo del reconocimiento de la identidad bicultural como fundamento original del estado-nación neozelandés. Como resultado de las políticas migratorias de las últimas cuatro décadas, Aotearoa Nueva Zelanda se ha convertido en uno de los países con mayor diversidad étnica en el mundo, iniciando una paulatina transición hacia el reconocimiento de una identidad multicultural emergente. La ponencia intentará presentar el rol de Aotearoa Latin American Community Incorporated en este proceso y sus esfuerzos por empoderar la comunidad latinoamericana como un actor determinante en la construcción de nuevas identidades a través de su programa de Desarrollo Comunitario y el proyecto "Travelling Sewing Box" o "Costurero Ambulante" creado por Victoria Martínez Azaro en colaboración con ALACINC.
\end{abstract}

Palabras clave: ALACINC - Multiculturalismo - Desarrollo Comunitario - Descolonizacion - Identidad - Nueva Zealanda - Aotearoa - E Tu Whanau Programme - Ministry of Social Development.

[Resúmenes en inglés y portugués en la página 49]

(1) Abogado de la Pontificia Universidad Javeriana con Magister en Derecho de la Universidad de Waikato Te Whare Wananga o Waikato. Posee más 7 años de experiencia en el sector comunitario y servicios sociales en Aotearoa Nueva Zelanda y actualmente se desempeña como Líder de equipo y Coordinador de Proyectos en Aotearoa Latin American Community Incorporated.

Los esfuerzos por consolidar un proceso de asentamiento de comunidades étnicas en Nueva Zelanda como respuesta a los flujos migratorios de los últimos cuarenta años en el país, tiene múltiples manifestaciones a través de políticas públicas y de iniciativas comunitarias protagonizadas por actores sociales, comunidades étnicas y líderes comunitarios.

Es así, que particularmente en el caso de Aotearoa Nueva Zelanda sus características históricas y culturales plantean condiciones particulares de asentamiento de minorías étnicas 
que incentivan procesos identitarios y sociales, con miras a generar comunidades más cohesionadas en el seno de una sociedad altamente diversa.

Los flujos migratorios, el desarrollo de la doctrina de los derechos humanos, la formulación de políticas multiculturales y la conquista de espacios comunitarios, económicos e institucionales por parte del pueblo indígena Maorí, y más recientemente por comunidades étnicas asentadas en el país como resultado de dichos flujos migratorios, confluyen en un acuerdo tácito que opera casi como un mantra. Es así, que la diversidad cultural se reconoce como un valor social, que define, enriquece y orienta la vida en común de todos los neozelandeses sin distinción, aunque no libre de expresiones de disentimiento generalmente asociadas con sectores xenofóbicos o supremacistas rechazados públicamente por la sociedad en su conjunto y por las autoridades máximas de su gobierno.

Aunque los fantasmas de la inequidad, la discriminación y la xenofobia permanecen, no hay duda en afirmar que la diversidad cultural es reconocida como un valor intrínseco y constituyente de la sociedad contemporánea neozelandesa.

Es en este contexto, que la labor de organizaciones e instituciones creadas y administradas por comunidades étnicas, así como la implementación de ciertos programas como lo veremos más adelante, cumplen un rol en la construcción de identidades, en un país altamente diverso producto de constantes olas migratorias, que pueden ser entendidos como ejercicios de descolonización en cuanto promueven diálogos interculturales que plantean alternativas al modelo hegemónico de prestación de servicios sociales en Nueva Zelanda.

\section{Antecedentes de un enfoque bicultural}

No podemos hablar de nuestro proyecto Travelling Sewing Box, del Programa de Desarrollo Comunitario y Terapia de Arte, y de la existencia de una organización como Aotearoa Latin American Community Incorporated, ALACINC, sin ofrecer un contexto de Aotearoa Nueva Zelanda.

En ese sentido, es importante reconocer que el estatus del Tangata Whenua, es decir del pueblo de la tierra (del Pueblo Maorí), es particularmente importante en el contexto de Estado Nación en Nueva Zelanda. Desde un principio, las relaciones entre las tribus Maoríes y la corona británica fueron diferentes a las relaciones que las potencias coloniales tuvieron con otros pueblos indígenas.

En efecto, la forma en que el proceso de colonización se dio fue de una manera distinta en donde, desde el comienzo, se establece su estatus independiente a través del reconocimiento de la declaración de independencia de las tribus maoríes de Nueva Zelanda $\mathrm{He}$ Wakaputanga o te Rangatiratanga o Un Tireni. Aunque los primeros contactos se dieron en el siglo XVIII, la coyuntura particular de la primera mitad del siglo XIX en el pacífico sur marcada por el proceso de colonización, la lucha por las rutas de comercio y los territorios en el pacífico sur entre la Corona Británica y el Imperio Francés. Las tribus Maoríes emiten su declaración de independencia en 1835 con apoyo de la Corona Británica con miras a afirmar su independencia, conservar su autonomía y gozar de la protección del Imperio Británico de agresiones de otras potencias coloniales. 
En 1840, cinco años después, se firma el Tratado de Waitangi Te Tiriti o Waitangi por un número importante de jefes, hombres y mujeres, de tribus Maoríes y la Corona Británica. Un tratado considerado por los historiadores como el hito fundacional de la Nueva Zelanda moderna, The Fabric of New Zealand.

No obstante múltiples controversias sobre el contenido del tratado debido a las discrepancias en sus versiones en lengua Inglesa y Maorí junto con el incumplimiento del tratado por parte de la Corona, múltiples confrontaciones armadas en la segunda mitad del siglo XIX y proceso de asimilación cultural resistidos por el Tangata Whenua incluso en nuestros días; el Tratado consolida la soberanía de la Corona Británica al tiempo que confirma un estatus especial de igualdad formal concedido a los Maoríes en relación con todos los súbditos de la Corona Británica. Un modelo único para las relaciones de la corona Británica con los pueblos indígenas alrededor del mundo en adición a los modelos implementados en Canadá, Australia y las trece colonias británicas en lo que hoy conocemos como Estados Unidos de América.

La Declaración de Independencia de las Tribus Maoríes de Nueva Zelanda y posteriormente el Tratado de Waitangi establecen un punto de partida para entender la identidad bicultural del Estado-Nación y la sociedad en Aotearoa Nueva Zelanda. Estos dos documentos consolidan formalmente el estatus especial del Pueblo Maorí Tangata Whenua hoy en día. El primero, porque reconoce la independencia y soberanía de las tribus independientes Maoríes de Aotearoa Nueva Zelanda y, particularmente el segundo, porque sirve como herramienta para afirmar la soberanía de la Corona Británica a cambio del reconocimiento de los Maoríes como súbditos de la Corona en igualdad de condiciones como cualquier otro súbdito británico, al menos en términos formales.

Te Tiriti o Waitangi afirma la soberanía de la Corona Británica sobre los territorios Maoríes a cambio del reconocimiento de la gente de la tierra Tangata Whenua como sus súbditos y la protección de sus tesoros, territorios y cultura, entre otros. De allí el enfoque bicultural en la construcción de la sociedad moderna de Nueva Zelanda que de ninguna forma podría considerarse libre de conflicto o dificultades.

Para la consolidación del contexto bicultural, fue necesaria la creación del Tribunal de Waitangi en 1975 con el propósito de investigar las violaciones de la Corona a sus obligaciones derivadas del Tratado de Waitangi. A esta iniciativa, le siguieron el desarrollo de los principios del tratado tanto por instancias del poder legislativo como por parte de las cortes del poder judicial, incluyendo las decisiones del Tribunal de Waitangi, con el objetivo de contribuir a la comprensión, interpretación y aplicación práctica del texto del Tratado en Te Reo Maorí y Lengua Inglesa, (Waitangi Tribunal) que trajeron como resultado un mejoramiento de las condiciones materiales de igualdad del Tangata Whenua.

\section{Flujos migratorios y una respuesta desde la multiculturalidad}

Al tiempo que se reconfiguraba la identidad bicultural de la sociedad, mediante el reconocimiento de las deficiencias del acuerdo social y el diseño de medidas para efectuar cambios conducentes a honrar las obligaciones derivadas del Tratado de Waitangi y de 
los compromisos internacionales de Nueva Zelanda en materia de derechos humanos; se produjo un aumento del flujo migratorio hacia Nueva Zelanda durante las últimas tres décadas del siglo XX.

Refugiados provenientes de países bajo gobiernos militares como la dictadura de Pinochet en Chile, por conflictos internos en Centroamérica, el sudeste asiático o África; o, migrantes como resultado de procesos migratorios de carácter económico se sumaron a las comunidades migrantes de China, India y las islas del pacífico para transformar a Nueva Zelanda en un país diverso y multicultural. Auckland, la ciudad más habitada del país que reúne a más del $30 \%$ de toda la población (con casi 2 millones de habitantes) es tal vez una de las ciudades más diversas del mundo, convirtiéndose en el hogar de más de 200 comunidades nacionales practicando sus tradiciones culturales y costumbres religiosas. Su población de 1.5 millones de habitantes es un motor importante de desarrollo y un espacio para la implementación de un número importante de políticas multiculturales. Estas políticas que parten de una visión bicultural de la sociedad, cuya identidad está en permanente evolución, han trascendido a otros espacios de construcción identitaria para responder a los desafíos de las olas migratorias más recientes. Es así, como asistimos a una transición hacia una visión multicultural de la sociedad en la que tiene sentido la existencia de organizaciones como Aotearoa Latin American Community Incorporated o programas como el E Tu Whānau del Ministerio de Desarrollo Social.

\section{E Tu Whānau: Primer nivel de diálogo intercultural}

Para Bhikhu Parekh (2006), toda sociedad requiere para su supervivencia y funcionamiento unos acuerdos básicos sobre valores y prácticas que regulan la conducta de sus miembros y constituyen parte de la estructura moral de una sociedad. Esta estructura es activa o pasivamente interpelada por grupos minoritarios en esferas más reducidas o en aspectos individuales de la vida.

Los valores comunes a la sociedad se articulan en tres niveles para conformar la cultura pública de una sociedad. En un nivel Constitucional, representado por los derechos fundamentales y las obligaciones de los ciudadanos; en un nivel legal, donde las leyes regulan aspectos específicos de la vida en común; y, finalmente, en un nivel cívico, donde las relaciones entre los miembros de la sociedad, valores y prácticas constituyen la cultura cívica de la sociedad (Parekh, 2006).

Estos valores públicos operativos de la sociedad que constituyen la estructura moral primaria de la vida pública están interrelacionados, no son estáticos y cambian de acuerdo con las circunstancias y la comprensión que de ellos hacen sus miembros. Esta dinámica es mucho más intensa entre más diversa es la sociedad y responde a esa interacción entre los valores públicos y los valores personales de sus miembros.

En este sentido, el reconocimiento de la Diversidad Cultural como valor y derecho se constituye en un elemento cohesionador que orienta políticas públicas; facilita la participación activa de los miembros de la sociedad en la implementación de dichas políticas (Boston \& Callister, 2005); fomenta la conformación de instituciones comunitarias de base y pro- 
yectos de desarrollo social (Roorda \& Peace, 2009); fomenta el reconocimiento de nuevas identidades e identidades híbridas; $y$, contribuye a la formación de una identidad nacional que acepta la diferencia cultural y se nutre de ella. Es en este contexto que los ministerios de gobierno desarrollan sus políticas con un fuerte componente que busca honrar la diversidad cultural como valor social buscando la participación de la población en la planeación, diseño, consulta e implementación de dichas políticas, programas y servicios teniendo en cuenta las tradiciones y códigos culturales. Ejemplo de ello es el programa $E$ Tu Whānau del Ministerio de Desarrollo Social.

Este programa es desarrollado y liderado con el apoyo de un Grupo de Referencia que se encarga de su implementación en colaboración con las tribus y clanes, en coordinación con el gobierno central; $y$ de acuerdo con los siguientes principios:

- KŌRERO AWHI / Comunicación efectiva

- AROHA / Dar sin esperar nada a cambio

- MANA MANAAKI / Ser la mejor versión de sí mismo y reflejarlo en la familia

- WHAKAPAPA / Conocer de dónde vienes y dónde perteneces

- WHANAUNGATANGA / Conexión y apoyo

- TIKANGA /Hacer las cosas de la forma adecuada y de acuerdo a nuestros valores

Su propósito fundamental es ofrecer apoyo a las familias y comunidades para permanecer y vivir libres de violencia, bajo principios y metodologías críticas frente al proceso colonizador y de colonialización ideológica, permitiendo y fomentando la participación y empoderamiento de las tribus Maoríes en el diseño, implementación y administración del programa apoyado por el Ministerio de Desarrollo Social del Gobierno de Aotearoa Nueva Zelanda.

Aunque es creado e implementado por autoridades Maoríes para atender necesidades sociales y familiares del Tangata Whenua, también ofrece un espacio de autonomía, rico e inspirador a comunidades migrantes y refugiadas de orígenes étnicos diversos que buscan soluciones a factores sociales que impactan diferenciadamente su bienestar social sobre la base de fortalecimiento de la cultura, valores familiares, liderazgo, conexiones sociales, participación comunitaria, sentido de pertenencia y colaboración entre comunidades étnicas, buscando inclusión y cohesión de una sociedad altamente diversa.

En este contexto, se plantea un primer nivel de diálogo intercultural (carácter interinstitucional) en el que las organizaciones sociales de base, en este caso concreto el Aotearoa Latin American Community ALACINC, desarrollan actividades y programas destinadas a atender las necesidades particulares de la comunidad Latinoamericana durante su proceso de asentamiento en Aotearoa Nueva Zelanda, en el contexto de políticas públicas que reconocen en la diversidad cultural una oportunidad para fortalecer comunidades más cohesivas en torno al respeto y apreciación de la diferencia.

No obstante el énfasis del trabajo realizado por la ALACINC se encuentra en la prevención e intervención en casos de violencia dado el propósito original del programa $E \mathrm{Tu}$ Whānau; el enfoque holístico de la organización reflejado en los principios propios del programa y en conexión con la práctica social de la organización, permite situar a los individuos a los que sirve, en un contexto familiar, comunitario y social para identificar 
las necesidades sociales de los beneficiarios de sus servicios en forma universal teniendo en cuenta las costumbres, lengua e identidad cultural de la población y los principios de atención de la Familia.

ALACINC diseña e implementa sus programas y actividades con el propósito de proveer las condiciones más adecuadas para el asentamiento de la población Latinoamericana en el contexto social de un país que por un lado se reconoce en sus orígenes como una sociedad bicultural, pero que se encuentra en permanente transformación dada su realidad multicultural.

Este nivel de diálogo intercultural tiene múltiples manifestaciones y ejemplos, algunas veces con resultados más satisfactorios que otros, pero como en el caso del trabajo de intermediación de organizaciones étnicas de base como ALACINC, siempre tiene lugar con agencias del gobierno e instituciones del sector de servicios sociales, para atender las necesidades y apoyar adecuadamente a los beneficiarios de dichos servicios de una forma culturalmente apropiada.

\section{ALACINC, una experiencia de empoderamiento}

Aotearoa Latin American Community Incorporated ALACINC surge en 1996, fundada por refugiados de la dictadura de Pinochet en Chile llegados a Nueva Zelanda a partir de 1975. Este grupo fue el primero, y en este sentido pionero del programa de cuotas de refugiados implementado por Nueva Zelanda en colaboración con la Agencia de Naciones Unidas para el Refugiado.

Se estableció como una organización de base comunitaria para atender a la población Latinoamericana refugiada y migrante en Aotearoa New Zelanda, aunque provee servicios en beneficio de otras comunidades étnicas si se requiere. Su propósito es responder a los desafíos de comunidades refugiadas en Nueva Zelanda durante el proceso de asentamiento en el país y apoyar en lo posible las medidas del Estado en busca del cumplimiento de sus obligaciones internacionales en materia de derechos humanos y derecho de refugio. ALACINC reconoce en su texto fundacional la relevancia del Tangata Whenua, Tikanga Maori y Te Tiriti o Waitangi para identificar el contexto en el que su trabajo social, cultural y formativo tiene lugar para el adecuado asentamiento en Aotearoa Nueva Zelanda de la población migrante y refugiada proveniente de Latinoamérica.

ALACINC provee servicios sociales, culturales y formativos con un enfoque holístico que parte de la comprensión del ser humano en sus contextos individual, familiar, comunitario, y en sus dimensiones emocional, psicológica, religiosa, mental y física; y, que tiene su desarrollo, en el seno una sociedad particularmente diversa como la de Nueva Zelanda.

La construcción de la identidad, para $A L A C I N C$, tan importante dentro del proceso de asentamiento en el país, parte de la articulación de cuatro diferentes niveles de comprensión (Parekh, 2006):

a. aquello que comparte con otros miembros de la especie humana; 
b. aquello que comparte con otros miembros de la comunidad nacional (chilena, colombiana, mexicana, etc.) y regional (latinoamericana) con la que se identifican;

c. aquello que comparte con la sociedad neozelandesa mayoritaria; $y$,

d. finalmente, aquello que refleja su propia identidad y particularidad individual como individuos.

En la prestación de los servicios sociales, los beneficiarios de ALACINC son reconocidos como seres humanos que pertenecen al género humano a través de particularidades culturales distintivas, en este caso definidas por patrones culturales latinoamericanos y específicamente determinados por sus tradiciones dentro de comunidades nacionales de los países de la región ${ }^{2}$, que buscan sus expresiones propias en el contexto de una sociedad diversa como la neozelandesa.

La existencia y trabajo de ALACINC sólo se puede entender en un contexto particular, determinado por las políticas migratorias y sociales de inclusión del gobierno de Nueva Zelanda y por una comprensión particular de los fundadores y continuadores de la obra sobre la importancia de los procesos sociales comunitarios.

Estas dinámicas sociales en las que los procesos de construcción de identidad cumplen un papel fundamental a través de la preservación de la lengua, la promoción de la cultura, la conquista de espacios de decisión autónoma y de implementación de modelos de práctica social y salud mental inspirados en códigos culturales, han tenido lugar en el contexto del país de acogida antes y después de la aplicación de políticas multiculturales de respeto por la diferencia y en busca de la inclusión de las minorías étnicas como resultado del proceso migratorio en el país.

Las actividades, programas y servicios implementados por la comunidad latinoamericana desde su llegada al país y posteriormente a partir de la fundación de ALACINC en 1994 han coincidido, al menos durante los últimos diez años, con un aumento en la incidencia de políticas de inclusión de minorías étnicas a través de la consulta y participación directa en el diseño de programas de atención; o, en la administración y ejecución de recursos públicos para la prestación de servicios por y en beneficio de los miembros de minorías étnicas, en este caso de la comunidad Latinoamericana.

Este proceso tiene lugar entonces en el contexto de políticas que favorecen el diálogo intercultural, el empoderamiento de comunidades étnicas y el fortalecimiento del tejido social con efectos prácticos en la consolidación y cohesión de una sociedad cada vez más diversa. Este proceso refleja la paulatina transformación en la forma en que el Estado de Nueva Zelanda se relaciona con los grupos étnicos a través de la redistribución de recursos económicos y, hasta cierto punto, de carácter político materializando intentos genuinos de dar a grupos no dominantes mayor acceso a los recursos e instituciones del Estado (Kymlicka, 2009), una dinámica tradicionalmente adoptada por el gobierno de Nueva Zelanda en su relación con la gente de la tierra, Tangata Whenua, los Maoríes.

ALACINC presta sus servicios de una manera holística, segura, accesible y culturalmente apropiados, que permita el asentamiento de la comunidad Latinoamericana en el país. Busca a través de sus servicios, actividades y programas la consolidación de lo que Bhikhu Parekh denomina comunidad cultural (Parekh, 2006, p. 154), en la que es posible cultivar el sentido de pertenencia a dicha comunidad cultural mediante la consolidación de una 
identidad regional latinoamericana, enriquecida por una diversidad de identidades nacionales, y que constituye a su vez, una especie de interface que habilita el diálogo intercultural entre sus miembros y el resto de la sociedad.

El diálogo intercultural a un nivel institucional también se materializa por medio del reconocimiento que hace ALACINC al estatus del pueblo Maorí y de su cultura en la conformación de la sociedad moderna neozelandesa. La constitución misma de ALACINC no sólo reconoce el estatus especial del Tangata Whenua sino la relevancia del Tratado de Waitangi como un hito fundacional que caracteriza la sociedad de Nueva Zelanda en cuyo seno se da un diálogo entre la cultura mayoritaria Europea, la cultura Maorí y más recientemente la multiplicidad de otras culturas.

Este reconocimiento, tiene una aplicación práctica, mediante la adopción que hace $A L A$ CINC de un modelo de práctica social que permite identificar un segundo nivel de diálogo intercultural (Institución-operadores-beneficiarios) con efectos sobre los operadores de los servicios y programas, en este caso los profesionales (trabajadores sociales, psicólogos, trabajadores comunitarios) y la población beneficiaria dado el marco de trabajo adoptado por la institución.

Te Whare Tapa Wha, como modelo de práctica social para la prestación de servicios sociales y de salud mental, busca el reconocimiento del individuo en su dimensión espiritual, emocional, psicológica y física; y, su desarrollo vital en el seno de una familia, de una comunidad y de una sociedad.

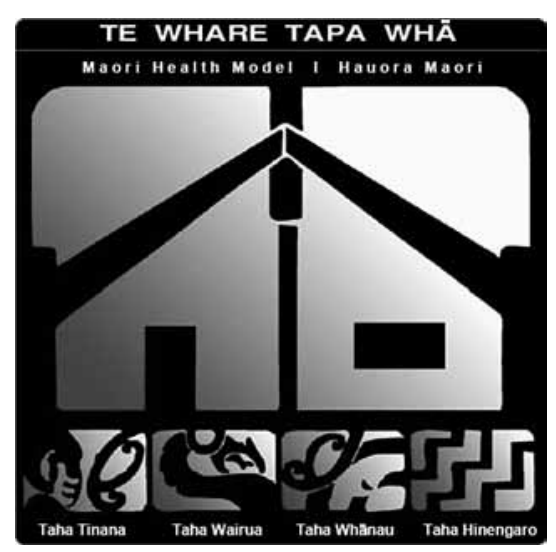

Figura 1. 
En efecto, este modelo de práctica social de ALACINC está inspirado en un modelo desarrollado con apego al Tikanga Maori, adaptado e implementado por ALACINC durante los últimos veinticinco años. La implementación de metodologías de trabajo dentro del modelo de práctica mencionado, incluye la consolidación de un proceso identitario Latinoamericano a través de la incorporación de los principios del Buen Vivir, la preservación de la lengua Castellana, la promoción de nuestra cultura y el reconocimiento de lenguas indígenas y tradiciones ancestrales a través de proyectos como la Biblioteca y Editorial Letras Latinas o grupos de baile y música tradicionales. En este sentido, el trabajo de la ALACINC y sus miembros intenta revisitar y contribuir con el proceso de construcción de identidad de la comunidad Latinoamericana en Aotearoa Nueva Zelanda, mientras presta servicios sociales con el propósito de proveer el apoyo necesario para el asentamiento apropiado de las familias migrantes y refugiadas provenientes de Latinoamérica.

Este proceso viene acompañado de una búsqueda por la igualdad. Una igualdad no sobre la base de la uniformidad cultural o religiosa, sino sobre el respeto de la diferencia. Igualdad en este caso involucra iguales oportunidades y libertades para ser diferente, un trato diferenciado teniendo en cuenta tanto las similitudes como las diferencias entre la comunidad Latinoamericana y los demás grupos étnicos articulados dentro de la sociedad neozelandesa, que garantice un tratamiento diferenciado pero equitativo. En este sentido, provee servicios sociales observando la idiosincrasia, la lengua, la cultura y los antecedentes de la población migrante y refugiada de la población, mayoritariamente Latinoamericana de habla castellana, articulando los estándares generales neozelandeses en materia de servicios sociales a sus necesidades particulares y características propias teniendo en cuenta su propia diversidad (Parekh, 2006, pp 239-240).

En la implementación de sus programas servicio y actividades, ALACINC busca no sólo la consolidación y desarrollo dinámico de una identidad Latinoamericana sino el acceso y ejercicio de los derechos en condiciones de igualdad a diferentes niveles. La igualdad en cuanto el respeto y ejercicio de los derechos, igualdad en cuanto a las oportunidades, igualdad en cuanto al ejercicio de poder y en cuanto a la protección ante la ley de una manera culturalmente apropiada que respete la diferencia. Este enfoque no es puramente teórico. Tiene una manifestación práctica en los servicios prestados por la organización permitiéndole responder efectiva, adecuada y empáticamente a las necesidades particulares de la población latinoamericana, caracterizada por una lengua, valores culturales, tradiciones religiosas e idiosincrasia diversos a las de las demás comunidades en el sociedad contemporánea neozelandesa.

La diversidad cultural como enfoque para la prestación de servicios e implementación de programas tiene varios implicaciones que mejoran la efectividad de los mismos y se relacionan con la naturaleza y alcance de la información suministrada a los usuarios; la adecuación de los servicios y programas de acuerdo a las necesidades y códigos culturales de los usuarios; el conocimiento, competencias culturales, habilidades especiales y atributos del personal que presta los servicios sociales a los usuarios; la variedad de servicios disponibles; $y$, la necesidad de implementar mecanismos de consulta para proveer espacios e participación a los beneficiarios de los servicios y así garantizar su consistencia y efectividad en tiempo real (Boston \& Callister, 2005). 


\section{Travelling Sewing Box o Costurero Ambulante: El diálogo de las fibras}

El programa de Desarrollo Social y Arte Terapéutico nació en 2016 para generar espacios de participación de la comunidad migrante y refugiada en arte, buscando incentivar comunidades mas resilientes; promover el uso del arte como medio de expresión narrativo de experiencias de migración y refugio que contribuyan en la construcción de procesos de identidad de la comunidad y sus individuos; incentivar la expresión artística de los participantes, la visibilización de sus voces; fomentar el bienestar familiar la sostenibilidad ambiental y económica; $y$, ofrecer espacios de emprendimiento social en beneficio de sectores vulnerable de la comunidad.

ALACINC adelantó varias fases en su ejecución, incluyendo consulta con la comunidad a través de encuestas y trabajo de base en los campamentos nacionales (2016 y 2017); trabajo colaborativo con organizaciones como Earth action Trust (Organización ambiental) y Shama (organización étnica para la atención y prevención de la violencia doméstica); y, la participación de representantes de tribus Maoríes expertos en técnicas textiles y el Protocolo de Harakeke que ofrecieron las condiciones para que un proyecto como el Costurero Ambulante/Travelling Sewing Box tuviera lugar.

El costurero ambulante como proyecto original de Victoria Martínez Azaro se alinea con el programa y plantea una oportunidad para explorar el proceso identitario en el contexto de los viajes migratorios de los participantes a través de la técnica textil y una metodología de trabajo, desarrollada colaborativamente por la ALACINC y la creadora del proyecto ${ }^{3}$, con el propósito de responder a las necesidades particulares de la población refugiada y migrante con la que el proyecto trabaja.

El proyecto se implementó en tres locaciones diferentes (Hamilton, Auckland y Waiheke Island) y en tres sesiones en cada locación, para trabajar tres instancias del proceso migratorio en su proceso formativo de identidad explorando el pasado y origen de los participantes (¿De dónde venimos?); el presente, el lugar de asentamiento, la cultural Maorí y su relevancia para el refugiado y migrante (¿Dónde estamos?; y, el futuro como ejercicio de proyección personal (¿Para dónde vamos?).

Los participantes tuvieron la oportunidad de crear sus propias piezas textiles plasmando sus historias de migración y refugio con fibras naturales de Latinoamérica y de Aotearoa Nueva Zelanda como la Muka y el Harakake. Como resultado se crearon 27 piezas individuales que finalmente conformaron una narración colectiva de los viajes, desafíos, proceso identitarios y sueños de la comunidad Latinoamericana que fueron expuestas en la muestra de Arte Glances of Diversity/Miradas de la Diversidad en 2017 en Auckland y que hicieron parte de la muestra Pertenencias en Junio de 2019 en la sede del MAP, evento coordinado por la Doctora Marcia Veneziani y la Diseñadora Textil Ximena González Eliçabe.

Durante las sesiones de los talleres, mientras las participantes adelantaban su proceso reflexivo y de aprendizaje de las técnicas, se facilitó la construcción de las narrativas individuales por parte de las participantes incorporando, en la segunda sesión, la presencia y formación en el protocolo de Harakeke para la extracción y uso de la fibra por parte de expertas tejedoras Maoríes que contribuyeron invaluablemente con el proceso no sólo identitario pero reflexivo de las participantes. 
En este punto se dio un diálogo intercultural entre los participantes y las maestras Maoríes de carácter directo y práctico. Un diálogo entre individuos no mediado por políticas para la prestación de servicios sociales o modelos de práctica social, sino de manera directa. Ese diálogo intercultural directo o de tercer nivel como parte de la construcción de las experiencias narrativas, tuvo lugar a través de las fibras mismas y las técnicas para su extracción, planteando procesos reflexivos y de comprensión muy profundos incluso de carácter emocional que potenciaron el trabajo de las participantes, fortalecieron sus propios proceso identitarios y permitieron establecer una conexión muy importante de doble vía con la cultura y el pueblo Maorí reconocido por las maestras Maoríes que participaron del proyecto (Vidal, 2017).

El reconocimiento de las similitudes y diferencias frente al otro ocupa un elemento fundamental en la definición de la identidad. El diálogo de las fibras y a través de las fibras que se dio durante los talleres despertó conexiones ancestrales entre los miembros de la comunidad latinoamericana y el Tangata Whenua, generando un lazo fundamental para los participantes en su propio proceso de asentamiento y construcción de identidad.

\section{Conclusión}

Aotearoa Nueva Zelanda plantea un espacio de construcción de identidad para la comunidad Latinoamericana caracterizado por una visión bicultural en transición hacia una visión multicultural de la sociedad de acuerdo con los antecedentes históricos del proceso colonizador $y$, con posterioridad a éste, como resultado de procesos migratorios durante los últimos 40 años.

ALACINC actúa como una interface que facilita el diálogo intercultural en tres niveles de operatividad, a nivel institucional (diálogo intercultural de primer nivel); a un nivel institucional-operador-beneficiario (diálogo intercultural de segundo nivel); $y$, a un tercer práctico entre individuos (diálogo intercultural de tercer nivel) que facilita el proceso de construcción de la identidad de la comunidad Latinoamericana, de identidades híbridas de sus miembros y de la construcción de la identidad nacional de Nueva Zelanda sobre la base de la Diversidad Cultural como uno de los valores fundantes de la sociedad contemporánea.

El trabajo de la ALACINC encuentra en E Tu Whanau un espacio creativo para la construcción de la identidad de la comunidad y la prestación de servicios sociales de manera diferenciada. Parte del empoderamiento de sus propios miembros con el propósito de atender las necesidades de la comunidad de acuerdo con metodologías y códigos culturales propios, respetando la diferencia y contribuyendo a través de ella con el proceso de construcción de una sociedad multicultural. En este contexto, el diálogo intercultural planteado también intenta descolonizar las metodologías al reconocer la relevancia de los principios Maoríes, el Tikanga Maori y su conocimiento ancestral, al tiempo que permite iniciar un proceso propio de carácter institucional y comunitario que permita la implementación de metodologías de trabajo que reflejen un conocimiento ancestral latinoamericano. 


\section{Notas}

1. Ponencia presentada en el Coloquio Pertenencias. Narraciones textiles de mujeres migrantes en el hemisferio Sur, organizado como parte de la muestra Pertenencias en la ciudad de Buenos Aires entre Junio y Septiembre de 2019.

2. Misión y visión de la Aotearoa Latin American Community Incorporated. www/Facebook/alacincnz

3. Metodologías de enseñanza práctica tradicional fueron alternadas con metodologías de Educación experiencial (Adventure-based counselling) y metodologías de trabajo social con población vulnerable.

\section{Referencias Bibliográficas}

Boston, J. and Callister P. (2005). Diversity and Public Policy. Policy Quarterly (Volume 1, Number 4,). Recuperado de: https://webcache.googleusercontent.com/ search?q=cache:MAF_sfO64EEJ:https://ojs.victoria.ac.nz/pq/article/download/4700/4 $184 /+\& \mathrm{~cd}=1 \& \mathrm{hl}=\mathrm{en} \& \mathrm{ct}=\mathrm{clnk} \& \mathrm{gl}=\mathrm{nz} \& \mathrm{client}=$ safari

E Tu Whanau del Ministerio de Desarrollo Social de Nueva Zelanda incluye los siguientes conceptos: Te mana kaha o te whānau! / La fortaleza y el poder de la familia; Tikanga / Cultura y valores tradicionales; Kahukura / Liderazgo; Nuestras historias; Liderazgo comunitario; e, Inclusión. Recuperado de: http://etuwhanau.org.nz

Kymlicka, W. (2009). Multicultural Odysseys. Navigating the New International Politics of Diversity. Oxford University Press

Misión y visión de la Aotearoa Latin American Community Incorporated. www/Facebook/ alacincnz

Parekh, B. (2006). Rethinking Multiculturalism. Cultural Diversity and Political Theory. Palgrave McMillan (2 Ed.)

Roorda, M. and Peace, R. (2009). Challenges to Implementing Good Practice Guidelines for Evaluation with Māori a Pākehā Perspective. Ministry of Social Development, Social Policy Journal, Issue 34.

Simon-Kumar, R. (September 5, 2019). The Multicultural Dilemma: Amid Rising Diversity and Unsettled Equity Issues, New Zealand Seeks to Address Its Past and Present. Migration Policy Institute. Recuperado de: https://www.migrationpolicy.org/article/rising-diversityand-unsettled-equity-issues-new-zealand

The principles of the Treaty of Waitangi as expressed by the Courts and the Waitangi Tribunal. He Tirohanga o Kawa Ki Te Tiriti o Waitangi, A Guide to the Principles of the Treaty of Waitangi as Expressed by the Courts \& the Waitangi Tribunal. Recuperado de: https://www.waitangitribunal.govt.nz/assets/Documents/Publications/WT-Principlesof-the-Treaty-of-Waitangi-as-expressed-by-the-Courts-and-the-Waitangi-Tribunal.pdf

Vidal, S. (2018, September 16). ALACINC Travelling Sewing Box [Video file]. Recuperado de: https://www.youtube.com/watch? $\mathrm{v}=\mathrm{krSaO}$ CoGk\&list=UUM2FMgslVilXjNkdm MNfDnA\&index $=11$ 
Abstract: Aotearoa New Zealand is one of the countries where the implementation of multicultural policies have contributed with processes of descolonisation departing from the acknowledgement of bicultural identities as the original fabric or New Zealand Nation-Estate. As a result of migratory policies of last four decades, Aotearoa New Zealand has become in one of the most ethnically diverse country of the world initiating a progressive transition towards a raising multicultural identity. This paper will attempt to depict the role of Aotearoa Latin American Community Incorporated in this process and its efforts to empower Latin American community as a prominent driving force in the construction of new identities through its Community Development Programme and the Travelling Sewing Project, crated by Victoria Martínez Azaro and implemented in collaboration with ALACINC"

Keywords: ALACINC - multiculturalism - Community Development - Descolonisation - Identity -New Zealand - Aotearoa - E Tu Whanau Programme - Ministry of Social Development.

Resumo: Aotearoa (nombre nativo para Nova Zelândia) é um dos exemplos onde a implementação de políticas multiculturais vem contribuindo no processo de descolonização partindo do reconhecimento da identidade bi cultural como fundamento original do estado nação neozelandês. Como resultado das políticas migratórias das últimas quatro décadas, Aotearoa (ou Nova Zelândia) tornou-se um dos países de maior diversidade étnica do mundo, iniciando uma gradual transição para o reconhecimento de uma emergente identidade multicultural. O trabalho apresentará o rol da Aotearoa Latin American Community Incorporated, e seus esforços para empoderar à comunidade latino-americana como um ator determinante na construção de novas identidades mediante o programa de Desenvolvimento Comunitário e o projeto «Travelling Sewing Box» criado por Victoria Martínez Azaro em colaboração com ALACINC.

Palavras chave: ALACINC - Multiculturalismo - Desenvolvimento Comunitário - Descolonização - Identidade - Nova Zelândia - Aotearoa - E Tu Whanau Programme - Ministry of Social Development.

[Las traducciones de los abstracts fueron supervisadas por el autor de cada artículo] 